\title{
iVirus: facilitating new insights in viral ecology with software and community data sets imbedded in a cyberinfrastructure
}

\author{
Benjamin Bolduc ${ }^{1}$, Ken Youens-Clark ${ }^{2}$, Simon Roux ${ }^{1}$, Bonnie L Hurwitz ${ }^{2}$ \\ and Matthew B Sullivan ${ }^{1,3}$ \\ ${ }^{1}$ Department of Microbiology, The Ohio State University, Columbus, OH, USA; ${ }^{2}$ Department of Agricultural \\ and Biosystems Engineering, University of Arizona, Tucson, AZ, USA and ${ }^{3}$ Department of Civil, \\ Environmental and Geodetic Engineering, The Ohio State University, Columbus, OH, USA
}

\begin{abstract}
Microbes affect nutrient and energy transformations throughout the world's ecosystems, yet they do so under viral constraints. In complex communities, viral metagenome (virome) sequencing is transforming our ability to quantify viral diversity and impacts. Although some bottlenecks, for example, few reference genomes and nonquantitative viromics, have been overcome, the void of centralized data sets and specialized tools now prevents viromics from being broadly applied to answer fundamental ecological questions. Here we present iVirus, a community resource that leverages the CyVerse cyberinfrastructure to provide access to viromic tools and data sets. The iVirus Data Commons contains both raw and processed data from 1866 samples and 73 projects derived from global ocean expeditions, as well as existing and legacy public repositories. Through the CyVerse Discovery Environment, users can interrogate these data sets using existing analytical tools (software applications known as 'Apps') for assembly, open reading frame prediction and annotation, as well as several new Apps specifically developed for analyzing viromes. Because Apps are web based and powered by CyVerse supercomputing resources, they enable scalable analyses for a broad user base. Finally, a use-case scenario documents how to apply these advances toward new data. This growing iVirus resource should help researchers utilize viromics as yet another tool to elucidate viral roles in nature.
\end{abstract}

The ISME Journal (2017) 11, 7-14; doi:10.1038/ismej.2016.89; published online 15 July 2016

\section{Viral metagenomics: an ecological tool with increasing impact}

Since the first viral metagenomic study was conducted in marine systems over a decade ago (Breitbart et al., 2002), the field has now expanded to include ecological studies of viral communities throughout the oceans globally, as well as diverse lakes and eukaryote-associated samples, including humans (Djikeng et al., 2009; Roux et al., 2012; Stern et al., 2012; Hurwitz and Sullivan, 2013; Hannigan et al., 2015). Highlights of some of the ecological advances enabled by these studies include revealing

Correspondence: BL Hurwitz, Department of Agricultural and Biosystems Engineering, University of Arizona, 1177 E 4th Street, Shantz Building, Room 403, Tucson, AZ 85721-0038, USA.

E-mail: bhurwitz@arizona.edu

or MB Sullivan, Department of Microbiology, Civil, Environmental and Geodetic Engineering, The Ohio State University, Riffe Building, Room 914, 496 W 12th Avenue, Columbus, OH 43210, USA.

E-mail: mbsulli@gmail.com

Received 25 January 2016; revised 3 May 2016; accepted 10 May 2016; published online 15 July 2016
(1) that virus-encoded 'auxiliary metabolic genes' extend far beyond the photosynthesis genes known from cyanobacterial cultures (Sharon et al., 2011; Hurwitz et al., 2013, 2014b), (2) long-term co-evolutionary features between viruses and their microbial hosts in both the human gut (Minot et al., 2013) and the oceans (Hurwitz et al., 2014b) and (3) the ecological drivers of viral community structure throughout the Pacific Ocean (Hurwitz et al., 2014b) and global surface oceans (Brum et al., 2015).

Many technological advances have enabled these discoveries-including optimized sampling strategies specific for viruses (reviewed in Duhaime and Sullivan, 2012; Solonenko et al., 2013) and improvements in low-input library preparation methods and decreased sequencing costs (Caporaso et al., 2012; Reuter et al., 2015) - and this has led to a data deluge whereby analytical limitations now represent the major bottleneck for virome-enabled viral ecology as follows. First, the number and size of newly generated metagenomes necessitates large-scale data storage and compute needs that require the development of community-available infrastructures specialized to viral sequence data. Second, the lack 
of tools for analyzing these large-scale data sets requires development by programmers, who speak a different 'language' from researchers generating much of the data. This often results in published code in public repositories that can be difficult to install or use without computational training. Finally, finding and analyzing viral data sets for comparative metagenomics is laborious and time consuming as raw and processed viral metagenomic data sets are deposited across a diverse array of data repositories, such as Genbank (Benson et al., 1998), EMBL (Kanz et al., 2005), NCBI genomes project (Wheeler et al., 2003), Metavir (Roux et al., 2014) and VIROME (Wommack et al., 2012).

Together, these technological limitations impede researchers from applying new tools to their data or leave them dependent on outsourcing data analysis to those unfamiliar with the ecosystems being studied. To enable scalability and accessibility of viral ecology research, we developed iVirus, a collection of software and data sets leveraging the CyVerse cyberinfrastructure (formerly iPlant Collaborative), to provide users with free access to computing, data management and storage and analysis toolkits (Goff et al., 2011). Briefly, iVirus seeks to collect viral sequence data sets in its Data Commons, adapt preexisting metagenomic tools as software applications (referred henceforth as Apps) and develop new analytical capabilities within the CyVerse cyberinfrastructure. Together, these advances help consolidate cutting-edge tools and curated data sets to empower researchers seeking to incorporate viral ecology into their own work.

Here we summarize the current capabilities of iVirus and invite community feedback, through the protocols.io interface (described later), to allow us to improve iVirus so that it becomes an indispensable tool for ecologists seeking to include viruses in their studies.

\section{What is CyVerse and how does it help my research?}

CyVerse (Goff et al., 2011) is the National Science Foundation-funded platform that seeks to bring together biologists and computer scientists to solve 'big data' problems in biology. Within the CyVerse cyberinfrastructure, users conduct research by navigating the Discovery Environment to identify data sets from the Data Commons (next section) and conduct analyses using Apps. Apps are like a computer software program, except that they (1) have been preinstalled, (2) leverage large-scale CyVerse compute resources and (3) can be integrated within a larger data context and workflow. This can improve biological research in multiple ways. First, it helps minimize installation issues and local systems administration needs that often impede biological research. Second, Apps can be linked together to create analytical workflows where the output from one App is used as the input for the next, in a linear manner. Because users can select which App they use at each stage in the workflow, the user can copy and update new workflows as new analysis tools emerge. Third, Apps ensure reproducibility and validity of research studies because they can be encoded into versioned Apps, along with raw or processed example data sets directly from the author. CyVerse can also assign digital object identifier to Apps or data sets to allow longerterm digital preservation and citable referencing in research articles. Finally, because all Apps and their output are tied to the user's home directory in the CyVerse Discovery Environment, all data are collected in one place. This avoids the common problem of data being scattered among multiple systems (high-performance computing (HPC), personal/lab computer, cloud computing) because of system-level requirements for implementing myriad bioinformatics software used for modern metagenomic analyses.

CyVerse Apps can be built by any developer using any source programming language to create community-specific tools. Moreover, the developer can encode hardware or software requirements within the App to lessen the burden on the user in installing and implementing that App. Once an App has been developed it can be shared with the community through the CyVerse cyberinfrastructure by a request to the CyVerse team via a simple public submission form. Once an App is public it can be vetted by the research community via a 5-star rating system and feedback forms. Furthermore, community developers can refine an App by duplicating and modifying it, and then republish the new App for recognition and further vetting and use by the community.

For developers, the process of creating an App follows one of two routes. First, Apps can be developed using CyVerse API (application program interface) called AGAVE (http://agaveapi.co/) that provides the developer with simple commands to access input data and write logs and results to the CyVerse Data Store. The developer can use the API to specify computational requirements for running code on HPC resources at the Texas Advanced Computer Center that are integrated in the CyVerse cyberinfrastructure. This process allows the developer to match the code to HPC compute resources and circumvent difficulties that users might experience in installing and reusing the code on different systems. Alternatively, Apps can be deployed using Docker images (www.docker.com), where the code is packaged with additional software dependences and can be run on CyVerse Docker-dedicated servers. Docker's compute autonomy and portability alone have made Docker images a mainstay for releasing open source code to the user community (Merkel, 2014), in addition to traditional code repositories such as Github (https://github.com). CyVerse extends Docker by allowing developers to deploy 
Docker images on CyVerse compute resources and attach these images to easy-to-use, web-based Apps in the CyVerse Discovery Environment. This provides developers with a means to publish code in an accessible format to a growing research community and to gain feedback on its utility rather than be drowned in inquiries about installation minutia.

\section{Centralized viral metagenomic data resources in the iVirus Data Commons}

The CyVerse cyberinfrastructure provides a common ecosystem for data, big or small, by providing a mechanism for communities to share data through a Community Data Commons. The iVirus Data Commons leverages these CyVerse resources to make data sets accessible from the Pacific Ocean Virome (Hurwitz and Sullivan, 2013), Tara Oceans Virome (Brum et al., 2015), Southern Ocean Virome (Brum et al., 2016), Virsorter Curated Dataset (Roux et al., 2015a) and legacy viral data sets from the retired Cyberinfrastructure for Advanced Microbial Ecology Research and Analysis (CAMERA) project (Seshadri et al., 2007). Beyond these, viral data were also mined and hand-curated from Genbank's Sequence Read Archive (SRA; Benson et al., 1998) and
MG-RAST (Wilke et al., 2015). In total, the iVirus Data Commons now contains data from 73 projects, 1866 samples and 5.5 billion reads, including contigs assembled from 75 viromes and 121 viral genomes.

\section{iVirus Apps are geared to viral metagenomics and community ecology}

iVirus Apps are developed using protocols defined by CyVerse-either through the Agave API or Docker - with focus on those needed for viral metagenomics. One operational goal of iVirus is to collect and deploy the most commonly used tools for viral metagenome pipelines-from raw read processing to assembly and analysis (see overview in Figure 1, and use-case scenario presented in the next section). This includes tools for read quality control, assemblers adapted to different input read types and various tools for analyzing assembled viral sequence data.

Because iVirus exists within the CyVerse environment, Apps developed through other communitybased CyVerse efforts are also available to iVirus. These include Apps relevant for viral metagenomics such as read prefiltering for assembly, gene calling, taxonomic identification and sequence alignment tools. This list also includes microbial metagenomic

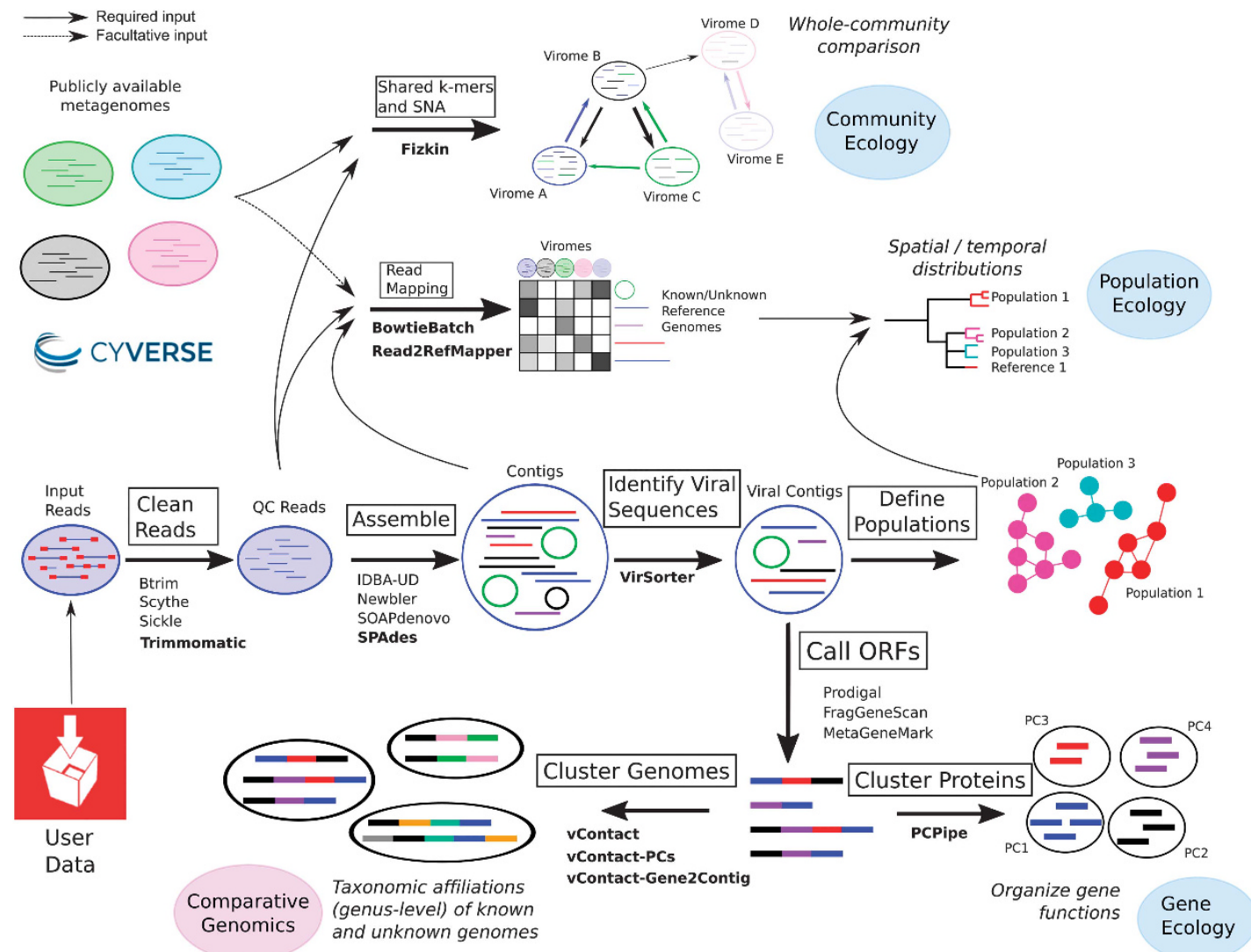

Figure 1 An organizational overview of how a user might leverage iVirus, iMicrobe and CyVerse Apps to analyze a viral metagenomic data set. Arrows indicate direction of use-case scenario. Processing stages are represented by text in blocks, with bold text indicating the Apps used in the use-case scenario. 
analysis Apps from iMicrobe that can be used to assemble contigs from metagenomes, predict and functionally annotate genes in these contigs and align them to each other and reference genomes (where available) for comparative genomic analyses. In general, Apps developed for HPC are located in a separate category within CyVerse, under 'High Performance Computing', whereas Docker and nonHPC-enabled Apps are organized into folders appropriate for their 'theme' (that is, iMicrobe, iVirus, Functional Analysis and so on). Because Apps are constantly being updated and developed, a full list of iVirus Apps are maintained at http://ivirus.us/avail able-tools/, along with computational protocols, App descriptions, relevant articles on tools and news in protocols.io at http://protocols.io/groups/ivirus.

iVirus is a subproject of iMicrobe and uses open source software developed by that project (http:// www.imicrobe.us and http://protocols.io/groups/ imicrobe) to query, search and download data in the iVirus Data Commons from a project-specific data website (http://data.imicrobe.us/). This webbased resource allows users to perform advanced searches on top of the iVirus Data Commons to discover viral data sets based on related metadata. To better enable search capabilities, iVirus metadata are mapped to the iMicrobe ontology that interconnects existing standards and terminology from the Minimal Information about any (x) Sequence Ontology (MIxS) and community-specific ontologies (BCODMO, ENVO, CheBI, BCO, OBOE). As such, the location of project specific data sets can be easily discovered and reused within CyVerse.

Beyond these more generally usable Apps, we have developed several iVirus Apps specifically for viral metagenomic study, with more being added as needs arise and development opportunities become available. In many cases, Apps developed for iVirus and iMicrobe also handle file manipulations, such as compression, separating reads and converting formats, so as to eliminate whenever possible the 'minor' details that are often time consuming and rate limiting for users.

A brief summary of selected iVirus Apps follows, along with reference to their source tools and how they have already enabled viral ecology where available. A broader analytical pipeline for processing viral metagenomes is overviewed in Figure 1 and described in a user-case scenario below and at https://www.protocols.io/view/Processing-aViral-Metagenome-Using-iVirus-ev3be8n.

\section{PCPipe}

This App compares open reading frames (ORFs) from a user-defined data set to existing viral protein clusters (PCs) as a means to organize proteins derived from viral metagenomics into functional units that can be used as (1) a universal functional diversity metric for viruses, (2) a scaffold for iterative functional annotations and (3) input to ecological comparisons through software such as QIIME (http:// qiime.org) (Caporaso et al., 2010). This is necessary as viral metagenomes are often dominated by novel sequences, where only $10-20 \%$ of reads map to known proteins in reference databases. In contrast, up to $50-70 \%$ of reads will typically map to PCs (Hurwitz and Sullivan, 2013). The PCPipe App accepts user-generated ORFs from viral metagenomic assemblies as input, matches them to ORFs in a user-supplied PC database and then self-clusters the remaining unclustered ORFs to capture the PCs unique to that data set. Reference sequences from new PCs are annotated using a collection of the nonredundant proteins and associated annotation from the SIMAP database (Rattei et al., 2009). PCs were originally developed for analyzing unknown proteins from the Global Ocean Survey that doubled the known protein universe at the time (GOS; Yooseph et al., 2007). This approach has proved similarly valuable for organizing viral protein sequence space (Hurwitz and Sullivan, 2013; Brum et al., 2015). Such an organizational tool has served as a means to estimate the size of the global virome at a few million proteins (Cesar Ignacio-Espinoza et al., 2013), as well as to make ecological inferences about viral communities with regard to their diversity (Roux et al., 2012; Hurwitz and Sullivan, 2013; Brum et al., 2015), niche differentiating genes (Hurwitz et al., 2014a) and ecological drivers (Brum et al., 2015).

\section{VirSorter}

This App identifies viral sequences in microbial genomes and metagenomic data sets (Roux et al., 2015a). This is necessary as viral genomes are underrepresented in databases-for example, 92\% of 1659 genome-sequenced phages derive from only 4 of 54 known bacterial phyla (Roux et al., 2015b). VirSorter can identify diverse viral sequences from microbial data sets, both integrated in the host chromosome and extrachromosomal. Briefly, VirSorter compares a data set of nucleotide sequences against a user-defined, precomputed viral database that includes viral sequences from RefSeq and (if desired) contigs assembled from viral metagenomes. The comparison also takes into account viral hallmark genes, as well as statistical enrichment of viral genes, depletion in hits to the PFAM database and strand bias. VirSorter output includes a summary file with 'confidence' categories for each identified sequence, as well as predicted proteins, PFAM domain hits, suspected circular sequences and metrics files. This tool is powerful and highly scalable-its first application was to nearly 15000 publically available archaeal and bacterial genomes, where VirSorter identified 12498 new hostassociated viruses and their genomes that augmented publicly available viral genome reference data sets by 10-fold (Roux et al., 2015b). Furthermore, VirSorter scales to handle contigs derived from metagenomic data sets (Roux et al., 2015a). VirSorter 
has since been used to identify viruses out of boiling hot springs in Yellowstone National Park (Munson-McGee et al., 2015), the Tara Oceans Viromes (Lima-Mendez et al., 2015) and hypersaline environments in the Atacama Desert (Crits-Christoph et al., 2016).

\section{vContact}

This App assigns contigs to taxonomic groups using the presence or absence of shared PCs along the length of the contig. This is critical as viruses lack a universal gene marker (Edwards and Rohwer, 2005) and $<0.1 \%$ of viruses in natural environments are represented in public databases (Brum et al., 2015), which necessitates new approaches to taxonomically classify surveyed viral genomes. Inspired by algorithms to detect prophage in microbial genomes (Lima-Mendez et al., 2008), vContact clusters contigs by their PC profiles (note: see preferred method for PC generation as vContact-PCs, but the user could generate PCs however they prefer). Reference sequences and their taxonomic lineages can be seeded within the analysis to improve clustering and taxonomic predictions. The vContact-generated network can be mined for its contig clusters (viral clusters) that roughly correspond to subfamily-level viral taxonomy. This approach has been used to organize nearly all (99.3\%) of the 12498 new viral sequences identified from publicly available microbial genomes into 614 'viral clusters', representing approximately genus-level groupings (Roux et al., 2015b). vContact can also incorporate annotations associated with contig and PCs, allowing users to examine the relationship of any annotated contig/PC in context of its vContact cluster.

\section{vContact-PCs}

This App serves as a companion tool for vContact to generate PCs using a Markov clustering algorithm (Enright et al., 2002). Users provide a BLASTP file of an all-against-all protein comparison, and vContact-PCs parses the BLAST file and applies Markov clustering algorithm against its similarity scores. vContact-PCs then exports files formatted for use with vContact.

\section{Fizkin}

This App performs Bayesian network analyses based on the amount of shared sequence content in viromes and relevant environmental metadata. Specifically, the App randomly subsets $300 \mathrm{~K}$ reads (or the lowest common denominator for reads in viromes) from up to 15 viromes and performs a pairwise all-vs-all kmer-based sequence comparison between all virome pairs as previously described (Hurwitz et al., 2014b). The kmer search in Fizkin is implemented in Jellyfish (Marcais and Kingsford, 2011) and is used to rapidly generate a matrix of shared sequence counts between each virome pair. This matrix is then used as input into a Bayesian network analysis (Hoff, 2005). The user can also input environmental metadata (continuous or discrete measurements, or latitude and longitude) that are used as part of the analysis to determine which environmental factors are significant in defining the structure of the network. Output includes: (1) a table indicating which environmental factors are significant and (2) a social network graph to visually represent the distance between viromes where statistical samples are taken from the marginal posterior distributions, and samples names are placed at the posterior mean. This type of social network analysis has been used to evaluate viral community structure and ecological drivers (Hurwitz et al., 2014b), as well as to quantify lysogeny through comparative analysis of experimentally induced and noninduced viromes (Brum et al., 2016).

\section{BatchBowtie}

This App runs bowtie2 on any number of reads files within a directory the user selects. Read recruitment against reference sequences (that is, viral genomes) can be employed as a means to visualize spatial or temporal distributions of genomes via the reads relative abundance across samples (Brum et al., 2015). The App additionally offers the ability to convert between interleaved and non-interleaved fastq files, compressed files and can generate SAM and BAM-formatted outputs (typically used by Read2RefMapper).

\section{Read2RefMapper}

This App consumes BAM alignment files (that is, from BatchBowtie), generating coverage tables and relative abundance plots-useful for identifying the abundance of reads against a set of reference sequences. Users can select a variety of filtering options based on the percent of read and reference covered (that is, $75 \%$ of a reference sequence must be covered to be considered 'present'), alignment identities as well as numerous coverage calculations. If users provide a file with the size of each metagenome, Read2RefMapper will also normalize the coverage between samples.

\section{Finding and using iVirus: a use-case scenario 'live' at protocols.io}

Taken together, the Apps mentioned above, in addition to the Apps already available in CyVerse, can be used to process a viral metagenome from 'raw' sequence to minimally characterized viral assemblies. The guide(s) for this and other examples are available at protocols.io: dx.doi.org/10.17504/proto cols.io.ev3be8n. These protocols are organized as collections, and available within the iVirus and 
iMicrobe groups at protocols.io, at https://www. protocols.io/groups/ivirus and https://www.proto cols.io/groups/imicrobe. These groups serve as a centralized location that offers additional documentation, feedback as well as citations using these tools and protocols. We have utilized protocols.io here so as to keep evolving processing steps up to date, as well as include images and annotations for each step. Furthermore, protocols.io is ideal for obtaining community feedback as it provides users the opportunity to ask questions and/or interact with the protocol's author through a simple to user interface.

The use-case scenario starts with test data that are reads from publicly available Ocean Sampling Day 2014 samples (https://github.com/MicroB3-IS/ osd-analysis/wiki/Guide-to-OSD-2014-data), a subset of which are already available on CyVerse data store. Next a user must first register for a free account in CyVerse (https://user.cyverse.org/) and then proceed through the process using available iVirus Apps summarized in Figure 1. Although only a few Apps are highlighted for each step, there are wide selections of choices for Apps available to the user. Example data are provided for each step in the iVirus Data Commons found under the /iplant/ home/shared/iVirus/ExampleData/ (https://de.iplant collaborative.org/de/?type = data\&folder = /iplant/home/ shared/iVirus/ExampleData) folder within the Cy Verse Discovery Environment. Output from each stage in this scenario is used as input for the next, though users can test any step individually, as each stage is organized separately and contains its own folders with inputs and outputs to help users identify which files are associated with each step.

\section{Step 1: upload read data}

Before processing can begin, reads to be analyzed must be uploaded to CyVerse's Data Store in the user's account. Small files can be uploaded from the user's computer through the Discovery Environment upload menu, with larger files transferrable through popular SFTP software, such as Cyberduck (https://cyberduck. io) and iRODS (http://irods.org). Data uploaded to a user's CyVerse home directory are private and accessible only to the user until they grant access to collaborators (via private invitation) or publish their data to the larger CyVerse community by transferring files to a shared Community folder. Users can also analyze or leverage growing publicly available data sets at iVirus in the Data Commons as previously described. Use case read files are already uploaded to the iVirus Data Commons.

\section{Step 2: quality control (QC) of read data using trimmomatic}

Protocol available at protocols.io: dx.doi.org/10. 17504/protocols.io.eygbftw.

Once raw read data have been uploaded, reads need to be quality filtered to ensure high-quality reads for assembly. FastQC (http://www.bioinfor matics.babraham.ac.uk/projects/fastqc/) is one such App already available through CyVerse and provides a visualization of read lengths, quality scores, duplicate reads, $\mathrm{N}$ and $\mathrm{GC}$ content of raw reads that can be used to determine the appropriate parameters for quality control. Once quality-filtering parameters have been determined for a given sequencing run via FastQC, reads files can be trimmed and quality controlled by using the Trimmomatic App.

Step 3: assembly of $Q C$ read data using SPAdes Protocol available at protocols.io: dx.doi.org/10. 17504/protocols.io.ewrbfd6.

Following QC, reads are then assembled using one of the assemblers available in CyVerse. Most frequently, assembler selection is based on read type (Sanger, 454, Illumina, PacBio and so on) and to a lesser extent, its performance for a particular sample type. IDBA-UD, SOAPDenovo, Trinity and SPAdes are available for viral metagenomic assembly. Some assemblers have high memory variants for larger data sets, and should only be used when the standard versions fail to assemble.

Step 4: identification of viral sequences from assembled data using VirSorter

Protocol available at protocols.io: dx.doi.org/10. 17504/protocols.io.eyjbfun.

To identify viral sequences from the contigs file, VirSorter is used. Generally, contigs $>3 \mathrm{~kb}$ can be successfully used as input-and can be single cell genomes, microbial or viral metagenomes, fragmented or complete genomes.

Step 5: characterizing viral sequence data through protein clustering with PCPipe and vContact Protocol available at protocols.io: dx.doi.org/10. 17504/protocols.io.eyhbft6.

Protocol available at protocols.io: dx.doi.org/10. 17504/protocols.io.ewabfae.

Large-scale characterization of viral genomic data remains one of the most daunting challenges in viral ecology. A relatively recent method of analyzing complex viral data is through organizing viral sequence space using PCs, reducing the problems associated with data complexity as a by-product. Regardless of the type of analysis, iVirus has access to a number of tools to characterize viral data.

\section{Concluding remarks}

Although viruses are increasingly recognized for their roles in microbial-dominated ecosystems, they remain understudied, particularly because of challenges stemming from the lack of centralized viral metagenomic resources. iVirus offers a community-focused resource, built on the CyVerse cyberinfrastructure and designed to directly address the challenges of viral ecology in the era of nextgeneration sequencing, HPC and big data analytics. 
This is done through (1) leveraging CyVerse Data Store to provide large data storage capacity and a centralized location for collecting data, (2) developing Apps, or software applications designed to take advantage of HPC resources that require limited bioinformatics training on part of the researcher, (3) collecting viral data sets in the iVirus Data Commons to provide a centralized location for discovering data sets via environmental metadata and collaborating within the field and (4) positioning these resources to maximize community exposure and feedback through extensive and 'live' documentation at protocols.io.

\section{Conflict of Interest}

The authors declare no conflict of interest.

\section{Acknowledgements}

We thank Joanne B Emerson, Dean R Vik, Gareth G Trubl, Consuelo Gazitua, Pilar Manrique and Jacob H MunsonMcGee for their helpful feedback in designing communityminded tools; Matthew Vaughn from the Texas Advanced Computer Center (TACC) and Nirav Merchant from the BIO5 Institute whose assistance in App development, troubleshooting and making Apps publicly available were invaluable. We thank Lenny Teytelman, Lori Kindler and Alexei Stoliartchouk for their agile development of protocols.io to accommodate requests for the iVirus group. The development of iVirus and this publication was partially funded in part by Gordon and Betty Moore Foundation Grants GBMF4491, GBMF4733, GBMF3305 and GBMF3790, by the US Department of Energy Office of Biological and Environmental Research under the Genomic Science program (Award DE- SC0010580) and by a TRIF award to the University of Arizona Ecosystem Genomics Initiative.

\section{References}

Benson DA, Boguski MS, Lipman DJ, Ostell J, Francis BF. (1998). GenBank. Nucleic Acids Res 26: 1-7.

Breitbart M, Salamon P, Andresen B, Mahaffy JM, Segall AM, Mead D et al. (2002). Genomic analysis of uncultured marine viral communities. Proc Natl Acad Sci USA 99: 14250-14255.

Brum JR, Hurwitz BL, Schofield O, Ducklow HW, Sullivan MB. (2016). Seasonal time bombs: dominant temperate viruses affect Southern Ocean microbial dynamics. ISME J 10: 437-449.

Brum JR, Ignacio-Espinoza JC, Roux S, Doulcier G, Acinas SG, Alberti A et al. (2015). Patterns and ecological drivers of ocean viral communities. Science 348: 1261498.

Caporaso JG, Kuczynski J, Stombaugh J, Bittinger K, Bushman FD, Costello EK et al. (2010). QIIME allows analysis of high-throughput community sequencing data. Nat Methods 7: 335-336.

Caporaso JG, Lauber CL, Walters WA, Berg-Lyons D, Huntley J, Fierer N et al. (2012). Ultra- high-throughput microbial community analysis on the Illumina HiSeq and MiSeq platforms. ISME J 6: 1621-1624.

Cesar Ignacio-Espinoza J, Solonenko SA, Sullivan MB. (2013). The global virome: not as big as we thought? Curr Opin Virol 3: 566-571.

Crits-Christoph A, Gelsinger DR, Ma B, Wierzchos J, Ravel J, Davila A et al. (2016). Functional interactions of archaea, bacteria and viruses in a hypersaline endolithic community. Environ Microbiol 18: 20642077.

Djikeng A, Kuzmickas R, Anderson NG, Spiro DJ. (2009). Metagenomic analysis of RNA viruses in a fresh water lake. PLoS One 4: e7264.

Duhaime MB, Sullivan MB. (2012). Ocean viruses: rigorously evaluating the metagenomic sample-to-sequence pipeline. Virology 434: 181-186.

Edwards RA, Rohwer F. (2005). Viral metagenomics. Nat Rev Microbiol 3: 504-510.

Enright AJ, Van Dongen S, Ouzounis CA. (2002). An efficient algorithm for large-scale detection of protein families. Nucleic Acids Res 30: 1575-1584.

Goff Sa, Vaughn M, McKay S, Lyons E, Stapleton AE, Gessler D et al. (2011). The iPlant collaborative: cyberinfrastructure for plant biology. Front Plant Sci 2: 1-16.

Hannigan GD, Meisel JS, Tyldsley AS, Zheng Q, Hodkinson BP, SanMiguel AJ et al. (2015). The human skin double-stranded DNA virome: topographical and temporal diversity, genetic enrichment, and dynamic associations with the host microbiome. MBio 6: e01578-15.

Hoff PD. (2005). Bilinear mixed-effects models for dyadic data. J Am Stat Assoc 100: 286-295.

Hurwitz BL, Brum JR, Sullivan MB. (2014a). Depth-stratified functional and taxonomic niche specialization in the 'core' and 'flexible' Pacific Ocean Virome. ISME J 9: 472-484.

Hurwitz BL, Hallam SJ, Sullivan MB. (2013). Metabolic reprogramming by viruses in the sunlit and dark ocean. Genome Biol 14: R123.

Hurwitz BL, Sullivan MB. (2013). The Pacific Ocean Virome (POV): a marine viral metagenomic dataset and associated protein clusters for quantitative viral ecology. PLoS One 8: e57355.

Hurwitz BL, Westveld AH, Brum JR, Sullivan MB. (2014b). Modeling ecological drivers in marine viral communities using comparative metagenomics and network analyses. Proc Natl Acad Sci USA 111: 10714-10719.

Kanz C, Aldebert P, Althorpe N, Baker W, Baldwin A, Bates $\mathrm{K}$ et al. (2005). The EMBL nucleotide sequence database. Nucleic Acids Res 33: 29-33.

Lima-Mendez G, Faust K, Henry N, Decelle J, Colin S, Carcillo F et al. (2015). Determinants of community structure in the global plankton interactome. Science (80-) 348: 1262073 1-1262073 9.

Lima-Mendez G, Van Helden J, Toussaint A, Leplae R. (2008). Reticulate representation of evolutionary and functional relationships between phage genomes. Mol Biol Evol 25: 762-777.

Marcais G, Kingsford C. (2011). A fast, lock-free approach for efficient parallel counting of occurrences of k-mers. Bioinformatics 27: 764-770.

Merkel D. (2014). Docker: lightweight Linux containers for consistent development and deployment. Linux J 2014: 2.

Minot S, Bryson A, Chehoud C, Wu GD, Lewis JD, Bushman FD. (2013). Rapid evolution of the 
human gut virome. Proc Natl Acad Sci USA 110: 12450-12455.

Munson-McGee JH, Field EK, Bateson M, Rooney C, Stepanauskas R, Young MJ. (2015). Nanoarchaeota, their Sulfolobales Host, and Nanoarchaeota virus distribution across Yellowstone National Park hot springs. Appl Environ Microbiol 81: 7860-7868.

Rattei T, Tischler P, Götz S, Jehl MA, Hoser J, Arnold R et al. (2009). SIMAP-A comprehensive database of pre-calculated protein sequence similarities, domains, annotations and clusters. Nucleic Acids Res 38: 223-226.

Reuter JA, Spacek DV, Snyder MP. (2015). High-throughput sequencing technologies. Mol Cell 58: 586-597.

Roux S, Enault F, Hurwitz BL, Sullivan MB. (2015a). VirSorter: mining viral signal from microbial genomic data. PeerJ 3: e985.

Roux S, Enault F, Robin A, Ravet V, Personnic S, Theil S et al. (2012). Assessing the diversity and specificity of two freshwater viral communities through metagenomics. PLoS One 7: e33641.

Roux S, Hallam SJ, Woyke T, Sullivan MB. (2015b). Viral dark matter and virus-host interactions resolved from publicly available microbial genomes. Elife 4: e08490.

Roux S, Tournayre J, Mahul A, Debroas D, Enault F. (2014). Metavir 2: new tools for viral metagenome comparison and assembled virome analysis. BMC Bioinformatics 15: 76 .

Seshadri R, Kravitz SA, Smarr L, Gilna P, Frazier M. (2007). CAMERA: a community resource for metagenomics. PLoS Biol 5: 0394-0397.

Sharon I, Battchikova N, Aro E-M, Giglione C, Meinnel T, Glaser F et al. (2011). Comparative metagenomics of microbial traits within oceanic viral communities. ISME J 5: 1178-1190.

Solonenko SA, Ignacio-Espinoza JC, Alberti A, Cruaud C, Hallam S, Konstantinidis K et al. (2013). Sequencing platform and library preparation choices impact viral metagenomes. BMC Genomics 14: 320.

Stern A, Mick E, Tirosh I, Sagy O, Sorek R. (2012). CRISPR targeting reveals a reservoir of common phages associated with the human gut microbiome. Genome Res 22: 1985-1994.

Wheeler DL, Church DM, Federhen S, Lash AE, Madden TL, Pontius JU et al. (2003). Database resources of the national center for biotechnology. Nucleic Acids Res 31: 28-33.

Wilke A, Bischof J, Gerlach W, Glass E, Harrison T, Keegan KP et al. (2015). The MG-RAST metagenomics database and portal in 2015. Nucleic Acids Res 44: gkv1322.

Wommack KE, Bhavsar J, Polson SW, Chen J, Dumas M, Srinivasiah S et al. (2012). VIROME: a standard operating procedure for analysis of viral metagenome sequences. Stand Genomic Sci 6: 427-439.

Yooseph S, Sutton G, Rusch DB, Halpern AL, Williamson SJ, Remington K et al. (2007). The Sorcerer II global ocean sampling expedition: expanding the universe of protein families. PLoS Biol 5: 0432-0466.

(c) (i) $\odot$ This work is licensed under a Creative Commons Attribution-NonCommercialNoDerivs 4.0 International License. The images or other third party material in this article are included in the article's Creative Commons license, unless indicated otherwise in the credit line; if the material is not included under the Creative Commons license, users will need to obtain permission from the license holder to reproduce the material. To view a copy of this license, visit http://creativecommons.org/ licenses/by-nc-nd/4.0/ 